\title{
The Research on Interaction Design of App Based on User Experience-Take Design Program Practice of Time Bank App as an Example
}

\author{
Chang Fangyuan \\ Shanghai Publishing and Printing College \\ shang9c@126.com
}

Keywords: User experience, mobile phone App, research on users, interaction design, time bank

\begin{abstract}
With the social economy growing faster and becoming more extensive in both speed and scale, smart phone application software, namely App, has been increasingly recognized by the majority of users with the development of smart phones. In people's daily life, work, and learning, App has gradually become an important way for people to communicate each other on machines. This paper takes design program practice of time bank App as an example to study and analyze the user experience.
\end{abstract}

\section{Introduction}

Based on the theory of user experience, the research on interaction design of App is conducted using the systematical method of theoretical content of the user experience and the user research to create a more rational and healthier operation experience for the user. From the user experience theory to the design practice, a more specific design rule and design method on the user experience of the App interaction design can be derived.

\section{Smart Phone App User Experience Layer Model}

1) User experience analysis of smart phone APP interface design is researched by practical investigation, user experience mainly includes: instinct layer, behavior layer, and reflection layer.

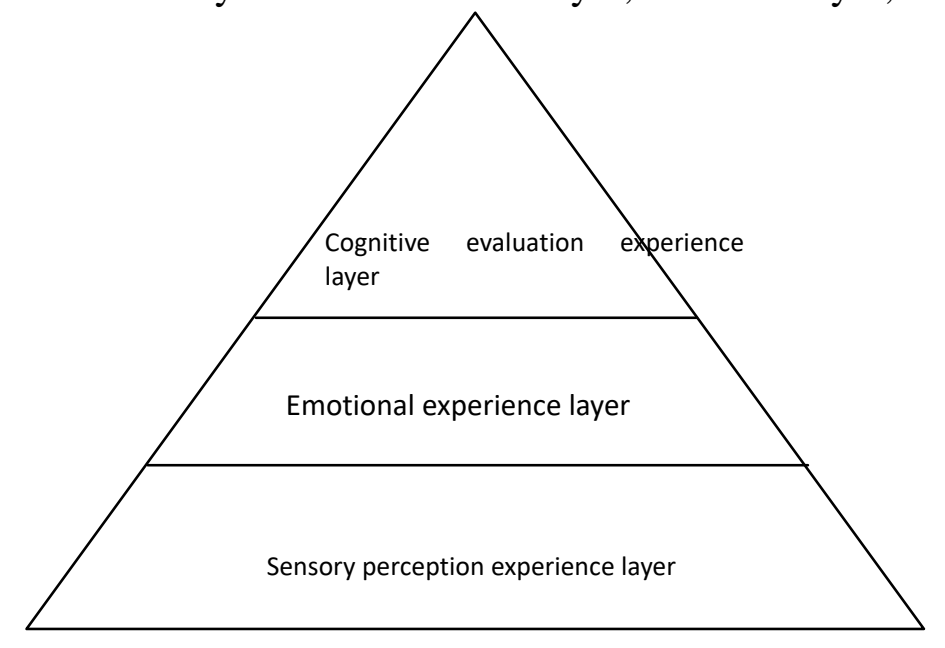

Figure 1 User Experience Layer Model

2) Design Layer

APP interface design is divided into functional interface and behavioral interface. The former uses the product logic to organize the interface to fulfill the function implementation. The latter uses the user behavior organization interface to emphasize the user experience. The relationship between the two is simultaneous, interactive, interdependent, and mutual integrative. At present, there is a lot of information on how to improve user experience and interface design. Many design specifications and theoretical rules set specifications for APP interface design, but the user experience for the 
product is inconsistent. It manifests in the application market for most APP design information disorder, layout complexity, inconsistency of visual and experience, intensive accumulation of element information, lack of effective guidance and visual guidance, non-standard page operations, imperfect feedback mechanisms, etc., which, gives the user a comfortable feeling.

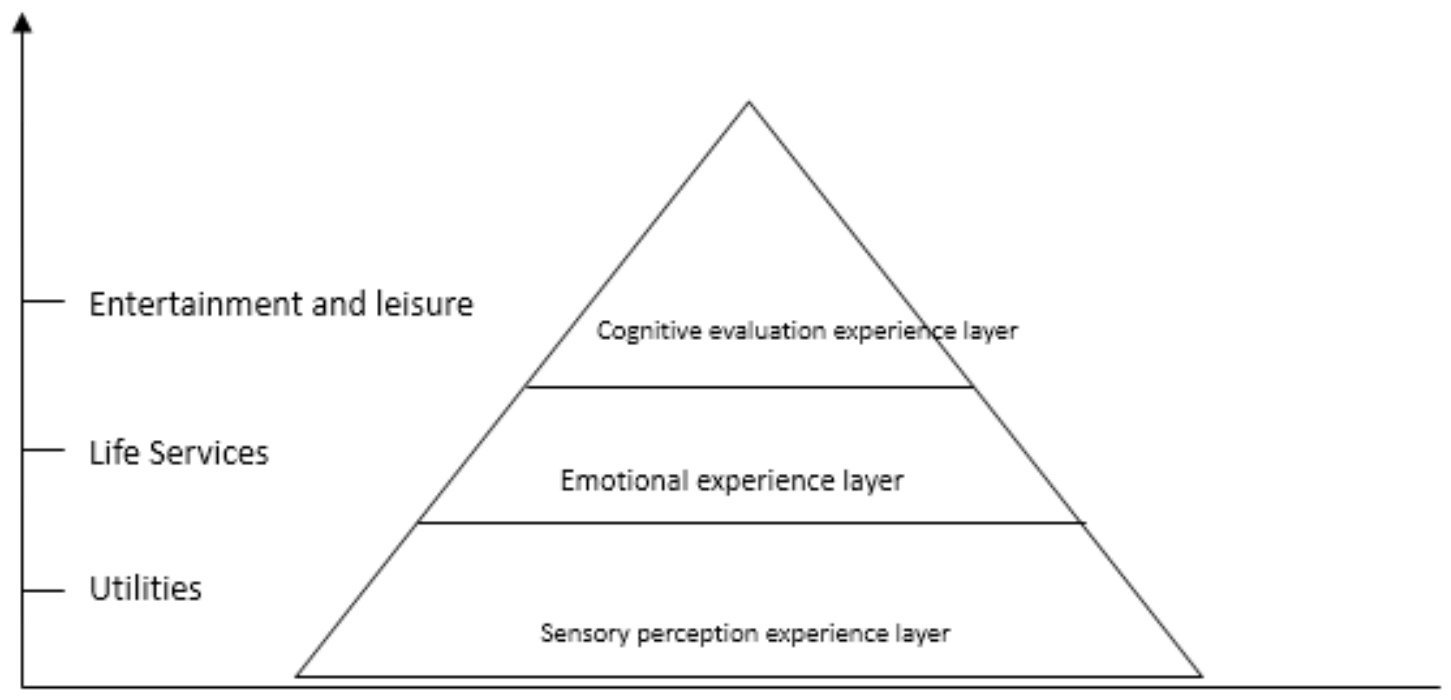

Figure 2 Determine the Position of the Product in the Model

\section{The Research on Interaction Design of App Based on User Experience}

\subsection{Content Positioning}

Content positioning also belongs to the scope layer, which is used to divide the scope of information content of Time Bank App. In terms of content positioning, the App will learn from the content of some mature foreign websites in the field of time bank, and based on this, it will combine the specific needs of domestic users to further make relevant content positioning.

\subsection{Interaction Design}

When it comes to product design, it is often the easiest to think of the product's structure, functions, materials, colors, etc. These elements make us have a more vivid understanding of the product.

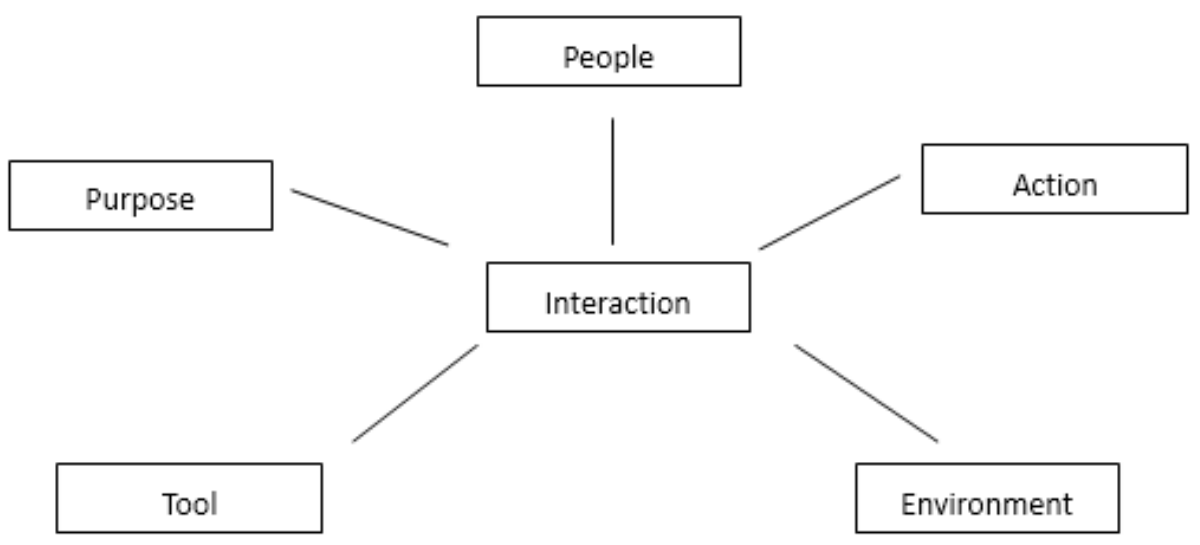

Figure 3 Five Elements of Interaction Design

However, these elements are only relatively independent features of the product itself. If the product designer only considers the product as a single individual, even if the product is used as a handicraft product for display purposes only, it may not be meaningful under certain circumstances. If the product and the people who use it are considered and seen as a whole, then the design of communication and interaction between products and people is crucial. A series of cores of 
interaction design are people, actions, necessary auxiliary tools for completing actions, surrounding environment and purpose, as shown in Figure 3.

\subsubsection{Interaction Model}

The interaction model is a concept of human-computer interaction. Its template derives from the extension of the five elements of interaction design. Simply put, on the one hand, the interaction model is based on the product's requirements document, and a series of performance charts that express the product's "external" ecosystem; on the other hand, based on the product's functional system, it analyzes the product's "inner" specific functions, and an information framework that embodies the flow of information and interactions between each function. First, it concatenates the five elements of interaction design into a simple basic format: people / by tools / finish actions / reach purposes / in the environment. It fills and correlates time bank App with these five elements: user/used time bank App client/operate/reach service activity purpose of resource sharing and message retrieval/in all environments and scenarios where it is possible to use mobile devices.

\subsubsection{Function System}

Through the interaction model, there is a more macro concept for Time Bank App. From the perspective of user behaviors studied by users, it starts from the user's factors and combines the five elements of interaction design, from the user's point of view to analyze the real needs contained in the analysis of the various functions behind the product. By the analysis of the interaction model, it is concluded that the functional system of the time-obscured App is mainly composed of an account system, a payment system, and a message system.

\subsubsection{Information Architecture}

The main object of the information architecture is information, through the design of the structure and organization, so that users can easily find and manage the purpose. In short, it is organizes and displays information reasonably to build a smooth bridge between information and users.

\section{Visual Design}

The visual design of an App is like the exterior design of a product design, whether it is a visual design or an exterior design, it is an intuitive experience to the user. the App's architecture, functions, and content are the same as the internal structure of the product, when the user first sees it, it will not have a lot of feelings about these, only in the process of use, will feel whether the function is reasonable and smooth and content is rich and effective or not. Therefore, the visual design of the App is as important as the exterior design of the product. This is the key to attract users to use it in the next step. Similarly, the visual design of App is also the same as the appearance of product design, and has many rules and methods. It is not a matter that designers can think unilaterally. Laws and methods should be followed in a series of styles, colors, icons, and layouts in the interface. Only in this way can a good user experience be designed for the App.

Prior to optimizing the interface design, the user's specific characteristics and behavior should be thoroughly studied and what the product needs shall be considered based on the user's perspective and psychology. Interface design is a cycle of repeated and continuous optimization process. It is necessary to study what the user thinks is a "meaningful question". According to the specific situation of the user, the interface design can be adjusted and modified to improve the use of the product. The excellent APP not only needs to satisfy the user in terms of basic use or practical functions, but also has an accurate design on the interface design nuances to impress people and enhance the feedback of user interaction and the direct operation experience. Excellent interface visual design can always bring good experience. The interface design should help the user to solve the problem comfortably and quickly with the correct goal orientation, reasonable product thinking, comprehensive design specifications and appropriate emotionalization so as to bring the unity of vision and experience to make design more invigorating users and more valuable. Determine the 
specific use groups for the nature and functions of the upcoming mobile phone APPs; secondly the guidance mode based on factors such as cognition and emotions is determined to ensure that the APP interface design constructed meets the needs of users; finally, the design direction is determined according to the user experience level model. The user experience model can determine the characteristics of the product, as shown in Figure 2. After determining the category of the product in the user experience layer model, it uses adjective description to further specify the product's characteristics.

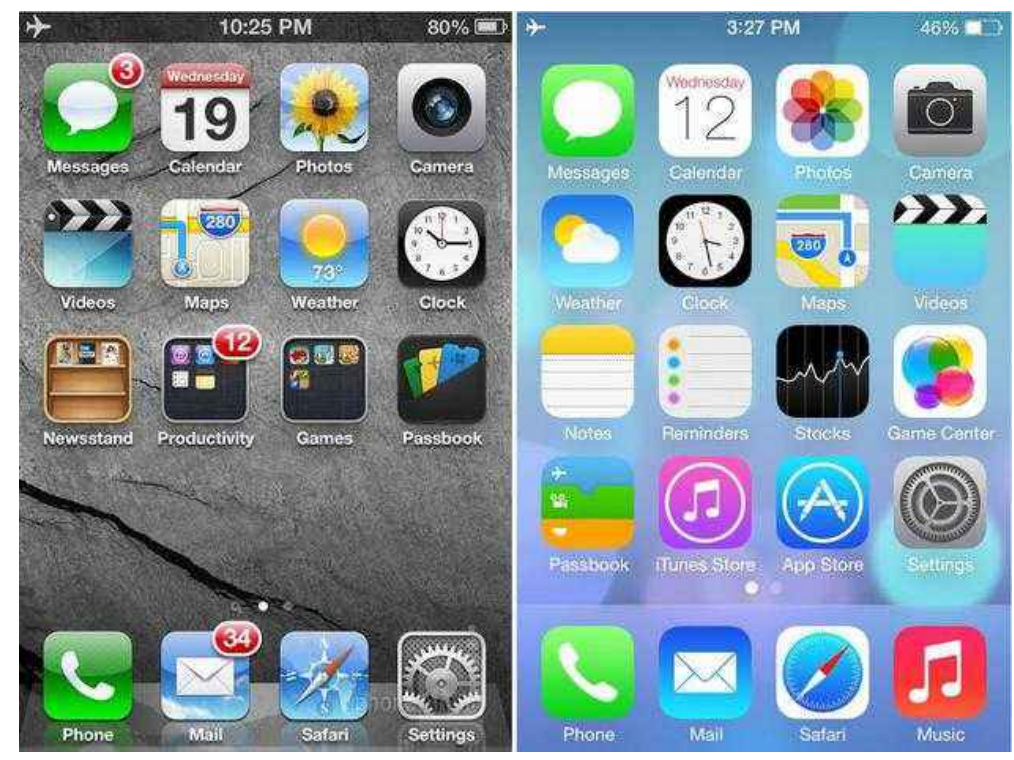

Figure 4 The Comparison of Flat Style and Simulation Style Interface

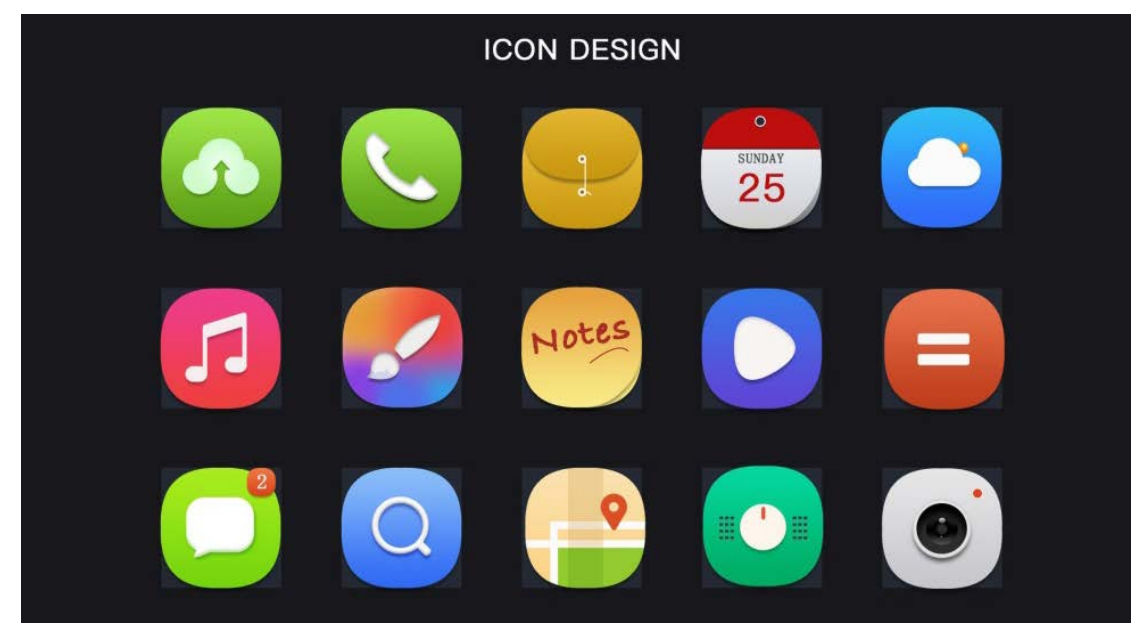

Figure 5 Flat Style App Diagram

\section{Conclusion}

The integration of user experience in APP design is a significant way to acquire more user groups. It should correctly recognize the necessity of user experience integration and further improve the interface design and content design of the equipment, so as to promote the improvement of its comprehensive competitive strength.

\section{References}

[1] Tang Lin, Chang Yu. The Research on User-centric Handheld Mobile Devices APP Interface Design[J]. Shandong Social Science, 2015(S2):97-99.

[2] Lin Li. Discussion on the Influence of User Experience on App Design[J]. Fujian Quality Management, 2016(03): 179 
[3] Xu Yanzhang. Design Thinking Model of APP Interaction Experience Based on "Internet+ "[J]. Art and Design (Theory), 2016,2(08):113-115

[4] Sun Haijiao. Research on APP Design Based on User Experience[J]. Art Education Research, 2017(08):69.

[5] Qi Mingyi. Exploring the Influence of User Experience on App Design[J]. Science and Technology Style, 2017(15):73. 